\section{rev Psi}

Revista de Psicología (UNLP)

https://revistas.unlp.edu.ar/revpsi

\title{
Acceso a la parentalidad en parejas diversas, 2018-2019
}

\author{
lara Vanina Vidal ${ }^{1}$ \\ Correspondencia \\ iaravaninavidal@gmail.com \\ Filiaciones institucionales \\ ${ }^{1}$ Facultad de Psicología, Universidad Nacional de \\ La Plata (UNLP, Argentina)
}

\section{Resumen}

Este artículo expone parte de las conclusiones de dos años de trabajo de investigación. El problema planteado refiere a la accesibilidad a la parentalidad en parejas diversas. Los objetivos propuestos eran: identificar cómo se accede a la parentalidad, por un lado, a partir de las técnicas de fertilización humana asistida garantizadas por la Ley n ${ }^{\circ} 14208$ $y$, por otro lado, mediante la adopción comprendida en la Ley no 26618 de Matrimonio Igualitario. Adoptamos un abordaje metodológico de estudio de caso, a través de entrevistas semi dirigidas a miembros del personal de Salud y del Poder Judicial. De esta manera se concluir que, frente a la situación actual en la Provincia de Buenos Aires, el marco normativo no resulta suficiente para el acceso, ya que la falta de insumos y de infraestrutura, así como las representaciones preexistentes de los actores intervinientes van en detrimento del cumplimiento de las leyes.

\section{Palabras clave}

accesibilidad | desigualdad | exigibilidad | familias diversas

\section{Cómo citar}

Vidal, I. (2021). Acceso a la parentalidad en parejas diversas, 2018-2019.

Revista de Psicología. HTtPs://DX.DOI. ORG/10.24215/2422572XE085

\section{Proceso editorial}

Recibido

23 jul. 2020

Aceptado

17 feb. 2021

Editor

Nicolás Alessandroni | Facultad de Psicología, Universidad Autónoma de Madrid (España)
ISSN

2422-572X

Licencia

Licencia de Cultura Libre CC-BY 4.0

(Compartir - Adaptar - Atribuir)

Entidad editora

RevPsi es una publicación de la

Facultad de Psicología (Universidad

Nacional de La Plata, Argentina) 


\section{Acesso à parentalidade em casais diversos, 2018-2019}

\section{Resumo}

Este artigo apresenta parte das conclusões de dois anos de trabalho de pesquisa. $\mathrm{O}$ problema levantado refere-se à acessibilidade da parentalidade em diversos casais. Os objetivos propostos foram: identificar como a parentalidade é acessada, por um lado, a partir das técnicas de fertilização humana assistida garantidas pela Lei 14.208 e, por outro, por meio da adoção da Lei 26.618 sobre Casamento Igualitário. Adotamos uma abordagem metodológica de estudo de caso, por meio de entrevistas semidirigidas com integrantes do pessoal do Poder Judiciário e de Saúde. Desta forma, pode-se concluir que, dada a situação atual na Província de Buenos Aires, o marco regulatório não é suficiente para o acesso, visto a falta de insumos e infraestrutura, bem como as representações pré-existentes dos atores intervenientes prejudicam o cumprimento das leis.

\section{Palavras-chave}

acessibilidade | desigualdade | força executória | famílias diversas

\section{Access to parenting in diverse couples, 2018-2019}

\section{Abstract}

This article presents conclusions of two years of research work. The research problem relates to the accessibility to parenting in diverse couples. The proposed objectives were: to identify how parenting is accessed, both thanks to assisted human fertilization techniques guaranteed by Law 14208 and adoption, as included in Law 26618 about Equal Marriage. We adopted a case study methodological approach and carried out semi-directed interviews with government employees, in the areas of Health and Justice. We concluded that, given the current situation in the Province of Buenos Aires, the regulatory framework is not sufficient to access parenting due to the lack of inputs and infrastructure, as well as pre-existing representations of the intervening actors which are detrimental to compliance with laws.

\section{Keywords}

accessibility | inequality | enforceability | diverse families 


\section{Aspectos destacados del trabajo}

- Se trabaja el acceso a la parentalidad en parejas diversas, encuadrada en las nuevas leyes.

- Se visibiliza la puesta en marcha de prácticas para la accesibilidad en el nuevo contexto.

- Se expone la desigualdad en el acceso a derechos que atraviesan las familias diversas.

- Se indaga acerca de los procesos de exigibilidad para el acceso por parte del contexto social.

El siguiente trabajo busca visualizar los resultados de una investigación llevada a cabo en la Facultad de Psicología, de la Universidad Nacional de La Plata, acerca del "Acceso a la parentalidad en parejas del mismo sexo y su incidencia con el contexto social y familiar", 2018-2019 (código S018). Dicho proyecto fue aprobado por la Secretaría de Investigación de dicha universidad, donde no fue un requerimiento de los organismos evaluadores y financiadores del estudio su aprobación por parte de un comité de ética.

En este artículo nos proponemos dar cuenta de cómo la aprobación y la puesta en forma de las leyes no suponen prácticas institucionales coherentes que las acompañen. Esto es de particular relevancia en países como la Argentina donde existen leyes de vanguardia con relación a los derechos de las personas diversas, pero que, por un lado, los actores y los profesionales que deberían llevar adelante el cumplimiento de dichas leyes proceden según su evaluación personal, y por el otro, si bien las normativas suponen presupuestos de política pública que acompañen, dichas condiciones mayoritariamente no se cumplen.

Luego, este artículo intenta responder al interrogante de cómo, tras 10 años de la Ley de Matrimonio Igualitario y de la Ley de Infertilidad Humana, el acceso a derechos es un proceso en el que se articulan de manera solapada condicionantes que tienen que ver con los dispositivos representacionales y materiales.

Este trabajo es el resultado de una investigación que buscaba indagar acerca de las condiciones del acceso a las técnicas de fertilización humana asistida por parejas diversas, que no cuentan con obra social ni con cobertura prepaga, propiciadas por la Ley 14208 (LRIHE 2010) en instituciones hospitalarias públicas de la Provincia de Buenos Aires. El segundo objetivo de la investigación fue visualizar la posibilidad de acceso a la parentalidad para las parejas diversas mediante la adopción.

Este artículo se organiza de la siguientemanera: en el primer apartado desarrollaremos antecedentes y normativa existentes; en el segundo presentaremos el abordaje metodológico utilizado; en el tercero se expondrán los resultados encontrados en 
las entrevistas; en el cuarto se compartirán las discusiones teóricas respecto de la problemática en relación con lo recolectado, y por último las conclusiones.

\section{Antecedentes y normativa: La Ley de Matrimonio Igualitario y las leyes de Reproducción Humana Asistida}

El objetivo del presente apartado es encuadrar la problemática dentro de un marco legal ya que pensamos las leyes como productoras de subjetividad, posibilitadoras de extensión de derechos y reguladoras a nivel social. Es importante que estas se puedan incorporar en la agenda de la sociedad, de los sujetos que en ella se construyen y desarrollan, y ser ejercidas por ellos. Describiremos la Ley 26.618 de Matrimonio igualitario y la Ley 14.208 de Fertilidad Asistida.

La sanción de la Ley n 26.618, más conocida como Ley de Matrimonio Igualitario, fue el corolario de una lucha de más de 30 años del movimiento LGBTTIQ junto a sectores sociales, políticos, culturales y académicos.

En cuestiones de género y sexualidad, quienes ingresaron primero a la escena política fueron las mujeres. En diversos espacios de movilización y reivindicación de derechos políticos, laborales y familiares, llevaron adelante movimientos sufragistas, anarquistas, socialistas y feministas. En este sentido, Fernández (2006) lo describe como tres movimie $\neg$ ntos: domésticos, políticos y académicos. Los primeros, en la esfera de lo privado en los años 50, gracias a los que empezaron a trabajar y a acceder a la escuela primaria y secundaria, se comenzaron a modificar los contratos conyugales y las nuevas modalidades de erotismo. La dimensión política de los movimientos feministas por la lucha en el plano de los derechos laborales y legales. Y el tercer movimiento de las académicas, a partir de los que empezaron, en los años 70, a cuestionar la ausencia de la dimensión de género en sus respectivas disciplinas.

Las mujeres fueron protagonistas de las luchas en diversos espacios en épocas de dictadura y de democracia en Argentina, desde las Madres de Plaza de Mayo durante la última dictadura militar, hasta nuestros días con todos los movimientos que impulsaron las leyes contra la violencia de género, a favor de la Identidad Sexual, así como la Ley de Matrimonio Igualitario.

Con la sanción de la ley, nuestro país se posicionó como el primero latinoamericano en reconocer a las personas de la diversidad sexual como sujetos de derecho. Esto marca un punto de quiebre, ya que el reconocer los derechos e implementar políticas de igualdad, inclusión y construcción de ciudadanía, posibilita que nuevos sectores sociales entren a formar parte de la agenda pública y pierdan el carácter de excluidos de los derechos que, hasta el momento de la sanción, parecían posibles sólo para las mayorías. La sanción de la ley, dirá Hiller (2010), genera un lugar de redefinición del espacio público que abre las puertas para la sanción de otras leyes que apuntan a la ampliación de derechos en nuestro país. Esta norma resulta entonces el antecedente de leyes, tales como la Ley n 26.743 de Identidad de Género, el Decreto 1006/2012 de Reconocimiento Igualitario, y la Ley n 26.862 , de Fertilización Asistida. 
La sanción de la Ley de Matrimonio Igualitario habilitó a que más de siete mil parejas contrajeran matrimonio dentro del territorio nacional. La puesta en práctica de dicha normativa, además del peso simbólico para los protagonistas, puso de relieve la legalidad de los vínculos familiares que incluían a niños y a niñas. Las parejas diversas, al contraer matrimonio, acceden a los mismos derechos que el resto de las uniones y con esto, logran suscribir a previsiones sociales, tomar decisiones de salud conjunta, decidir sobre la guarda de sus cuerpos, contar con derechos de herencia, de adopción y de inscripción conjunta de los niños y niñas. Estos últimos tienen los mismos derechos que otros niños y otras niñas, siendo legalmente inscriptos como hijos e hijas de la pareja y reconociendo su identidad familiar.

Abrir el debate con respecto a la parentalidad y la posibilidad de las parejas diversas de ser padres, conlleva la creación de representaciones, de valores y de modelos de comportamiento que construyan una cultura familiar donde las nuevas uniones estén comprendidas.

En esta línea entonces, podemos proponer a las políticas públicas de la mano de Pecheny y De La Dehesa (2010), como manifestaciones de aspectos instrumentales de lo político, pero también como dimensiones expresivas y comunicativas. Las políticas públicas como mensajes a la sociedad siguen una lógica expresiva propia, a su vez, de las políticas identitarias.

La Ley de Fertilización Asistida de la provincia de Buenos Aires $\mathrm{N}^{\circ} 14.208$, sancionada el 2 de julio de 2010, plantea que "se amplía el derecho a la salud a miles de parejas de la provincia de Buenos Aires y renueva las esperanzas para concretar el sueño de formar una familia". Sin embargo, prevé una serie de limitaciones, mujeres entre 30 y 40 años e informes ambientales que muchas veces se convierten en un obstáculo.

La ley postula en dos artículos que la condición para el tratamiento es la de ser una pareja heterosexual. En el primero, define la infertilidad, según la OMS, como "ausencia de embarazo luego de un año de vida sexualmente activa" (entre una mujer y un varón). El segundo artículo explicita que se autoriza la fertilización homóloga, es decir, con las propias gametas (óvulo y espermatozoides de la pareja, no donados).

Por su parte, la Ley Nacional no 26.862 de "Acceso integral a los procedimientos y técnicas médico-asistenciales de reproducción médicamente asistida”, se reglamentó el 19 de julio del 2013, y "establece que tienen derecho a las prestaciones de reproducción médicamente asistida todas las personas, mayores de edad, sin discriminación o exclusión de acuerdo a su orientación sexual o estado civil". De esta manera, quedarían saldadas varias limitaciones de la provincial, como son la edad y la orientación sexual. Sin embargo, en la práctica es la Provincia de Buenos Aires quien reglamenta los programas y, por ende, los presupuestos.

La Ley de Fertilización Asistida fue paralela a las discusiones de la Ley de Matrimonio Igualitario, sancionada el 15 de Julio del 2010. Si bien en la primera no estaba en discusión el tema de las parejas diversas, dicha problemática se puede visibilizar en 
los debates sobre el Matrimonio Igualitario. Hiller (2010) analiza el contexto socio político institucional que ha dado lugar al dictado de la Ley de Matrimonio Igualitario y remarca la incidencia de los movimientos feministas y LGTTBIQ para la puesta en discusión, en el espacio público, del debate de la Ley de Matrimonio Igualitario. A partir de entrevistas a integrantes de los movimientos, a diputados y a senadores, entre otros, Hiller pesquisó que el tema se impuso como de agenda pública y que los legisladores no pudieron obviar su tratamiento. Asimismo, rescató la importancia de las audiencias públicas realizadas en siete provincias (Chaco, Corrientes, San Fernando del Valle de Catamarca, San Miguel de Tucumán, Córdoba, San Juan, San Salvador de Jujuy y Mendoza) que de alguna manera traían las voces y opiniones de la "Argentina profunda", como lo solicitaban algunos diputados.

En este sentido, Fernández (2015) sostiene que los debates en los foros públicos sobre la Ley de Matrimonio Igualitario mostraron posicionamientos precarios y desinformados que no permitieron pensar más allá del prejuicio; $y$, como plantea Raíces Montero (2004), toda discriminación pública y privada contra las personas intersexuales afecta a la población en su conjunto, lo mismo podemos pensar sobre las parejas del mismo sexo.

Dentro de los debates de la Ley de Matrimonio Igualitario, se discutió acerca de su denominación. Compartimos con el jurista Etchichury (2010) la importancia de proponer como el título de Ley de "Matrimonio Igualitario" y no de "Unión Familia", u otro nombre, ya que se mencionaría como una institución gemela que llevaría los mismos derechos. El mismo nombre ha sido la clave de la ampliación de derechos.

Una de las preguntas que se hacen algunos psicoanalistas como Vidal (2010) es: ¿Por qué los homosexuales desearían hoy lo que los heterosexuales rechazan? La autora sostiene que es necesario cuestionar la familia para abrir a nuevas formas de lazo y no pensarla con parejas diversas, igual a la tradicional. Vidal menciona que es escéptica de pensar las familias diversas ajustada a los criterios de la familia patriarcal y propone adherir a la idea de Butler que apunta a pensar nuevas formas de lazo social que permitan una vida más vivible, vidas que merezcan ser vividas.

Precisamente, Butler (como se cita en Birulés, 2008) dice estar de acuerdo con el derecho al matrimonio homosexual pero que, si no se sigue luchando por las familias alternativas, los parentescos y los otros modos de asociación personal, no serán suficientes. Además, cuestiona la exigencia, de la comunidad LGTTBIQ, del reconocimiento de parte del Estado del matrimonio homosexual o sea de dicha relación, y se pregunta “¿para qué necesitamos que el Estado defina nuestras relaciones?”.

Los reclamos de los grupos de las organizaciones militantes que luchan por la igualdad de derechos de las llamadas minorías sexuales, como los estudios Validación de un cuestionario de riesgos psicosociales en el trabajo en población trabajadora argentina y los movimientos LGTTBIQ, representaban, antes de La Ley de Matrimonio Igualitario, la igualación y el derecho al matrimonio, como así también la posibilidad del ejercicio de la parentalidad a partir de la inscripción en el registro civil de los hijos/as con el apellido de ambos, entre otras demandas. En este 
sentido, las discusiones en las parejas homosexuales, a partir de la Ley de Matrimonio Igualitario, acerca de la decisión de qué apellido antecede al otro, instala la elección de quién sigue con la genealogía.

Hasta aquí, se presentaron los antecedentes de los estudios sobre la temática, así como las discusiones acerca de las normativas teniendo en cuenta lo que encuadran y garantizan. En el presente trabajo, intentaremos visualizar cómo inciden estas leyes, en el acceso a la parentalidad en parejas diversas. Si bien los estudios expuestos discuten acerca de las leyes, no hemos encontrado trabajos sobre cómo se llevan adelante las prácticas en la política pública, para que la normativa sea cumplida. Es decir, que se pueda acceder a TRHA de manera gratuita en hospitales públicos y que la adopción no sea obstaculizada para las parejas diversas. Por esta razón, sostenemos la relevancia de nuestra investigación, que viene a responder la pregunta de cómo se están efectivizando dichas leyes en la Provincia de Buenos Aires.

\section{Abordaje metodológico}

En este trabajo se adopta un abordaje cualitativo cuyo diseño está destinado a brindar una descripción del caso en la mencionada institución, a partir de entrevistas semidirigidas sobre la problemática seleccionada, y así cualificar el marco conceptual existente y trabajos previos del equipo (Strauss y Corbin, 1990).

\section{Instrumento metodológico}

De acuerdo al marco metodológico señalado se diseñaron y se realizaron entrevistas semidirigidas a profesionales del ámbito de la salud, del poder judicial y de ONG que trabajen sobre los derechos de la comunidad de lesbianas, gays, travestis, transexuales, bisexuales, intersexos, queer (LGTTBIQ), para indagar acerca de los procedimientos de acceso a derechos.

La entrevista contó con 20 preguntas abiertas, cuyo eje estaba divido en tres partes: en la primera, se indagaba acerca del conocimiento de familias diversas y su funcionamiento. En la segunda parte, sobre la noción de las leyes, su participación (desde la asesoría o la militancia) y su opinión al respecto. En la tercera parte, acerca de la existencia de consultas de parejas diversas y su resolución. Las preguntas de las entrevistas para los equipos de salud fueron las mismas y se diseñaron otras preguntas para el poder judicial, ya que la adopción contiene otras aristas.

Realizamos un role play entre los miembros del equipo para probar las preguntas diseñadas. Después de la primera entrevista realizada a un profesional de La Plata de manera presencial, se ajustaron las preguntas para realizar las otras dos que correspondían al equipo de salud.

\section{Participantes}

Realizamos tres entrevistas en el ámbito de la salud, a integrantes de tres de los cuatro hospitales públicos que realizan, por la ley, los Tratamientos de Reproducción 
Humana Asistida (TRHA) de manera gratuita. En el hospital San Martín de La Plata entrevistamos a dos médicas del equipo de Fertilidad, en el Penna de Bahía Blanca, a una profesional del Servicio de Fertilidad y en el Allende de Mar del Plata, a una médica. Realizamos una entrevista en el Poder Judicial, a la psicóloga del equipo del juzgado que cuenta con el Registro de Adopciones de la ciudad de La Plata. Además, entrevistamos a un abogada de una ONG que trabaja por los derechos de la comunidad LGTTBIQ.

Se tuvo en cuenta el criterio de accesibilidad, es decir, la posibilidad de convocar a dichas personas para ser entrevistadas. La técnica de bola de nieve fue elegida como medio de selección de la muestra. Esta consiste en que una de las personas entrevistadas genere un contacto con otra persona con las mismas características de las participantes (es una técnica de muestreo no probabilístico).

Solicitamos a las personas seleccionadas para la muestra, que prestaran su consentimiento informado y que aclaren que los datos obtenidos serían de carácter confidencial y que permanecerían sus nombres en el anonimato. En el primer momento de la entrevista, expusimos el objeto de la investigación para que la persona pudiera evaluar si deseaba continuar. Asimismo, aclaramos que intentaríamos que no pueda identificarse su nombre. Preguntamos si nos autorizaban a grabar las entrevistas para poder ser fieles a lo que se decía.

\section{Análisis de los datos}

A partir de la desgrabación y de la lectura de las entrevistas, se construyeron categorías de análisis. Se procedió entonces, a la categorización de cada una para posibilitar así la caracterización de las semejanzas y/o diferencias. De este modo, se comparó lo relevado con el marco referencial, lo cual permitió examinar y articular las coordenadas teóricas con la información que surgió de las entrevistas a las participantes incluidas.

Realizamos un análisis por tema, como lo proponen Blanchet y Gotman (1992), donde diseñamos una grilla de análisis para encontrar una coherencia temática entre las entrevistas. La identificación de temas y la construcción de la grilla de análisis se efectuaron a partir de los objetivos de la investigación, cualificados después de la lectura de las entrevistas, en una interacción entre el problema planteado y el corpus conceptual que se desarrolla en el apartado de discusión.

Los equipos de salud. La Ley de Matrimonio Igualitario habilitó desde el marco normativo que las parejas diversas tuvieran posibilidad de ser padres y madres (LNMI, 2010). En el seno de la investigación, realizamos en una primera fase, entrevistas a parejas del mismo sexo con proyectos de hijos/as y con hijos/as. En ese momento nos encontramos que, si bien existe la Ley Nacional de Reproducción Médicamente Asistida y la Ley Provincial de Reconocimiento de la Infertilidad Humana como enfermedad, en los casos donde no cuentan con obra social o con el dinero suficiente para llevar adelante la práctica en lo privado, el acceso a 
la parentalidad es casi imposible. En Argentina existen las obras sociales que son entidades privadas, dependientes de los sindicatos, que prestan atención médica a los trabajadores en relación de dependencia. Son uno de los componentes del sistema privado de salud en el país junto a la medicina prepaga.

En las entrevistas a parejas del mismo sexo con hijos/as, la mayoría habían realizado los tratamientos de manera privada y una de ellas por inseminación casera, es decir el procedimiento por el cual, a partir de la compra de la muestra de semen en un banco de donantes, se realiza la inseminación en el domicilio de la pareja sin que medie un equipo de salud. Una de las preguntas que nos planteábamos era saber que ocurría con aquellas personas, que no tuvieran los medios necesarios para pagar la muestra de semen o el tratamiento. Si bien la ley provincial exige gametos propios, lo que dejaría de lado a las parejas de mujeres, la nacional no exige que los gametos sean propios, es decir, se podría recurrir a un banco de esperma.

La ley provincial prevé que dichas prácticas se lleven a cabo de manera gratuita en cuatro hospitales públicos de la Provincia de Buenos Aires: el San Martín de La Plata, el Penna de Bahía Blanca, el Allende de Mar del Plata y el Güemes de Haedo. Por ello entrevistamos a integrantes de los equipos de tres de esos cuatro hospitales. Lamentablemente, no pudimos contactar al equipo del hospital Güemes.

En los inicios de la investigación, en abril de 2015, entrevistamos al equipo del hospital San Martín para interiorizarnos acerca de la situación. En el 2019, volvimos a entrevistar a miembros del mismo hospital y sumamos a integrantes del equipo del Penna y del Allende. En el primer contacto con el San Martín, dialogamos con las psicólogas y con la trabajadora social, ya que en ese momento fue imposible concertar un encuentro con la médica ginecóloga. En el segundo contacto, cambió parte del equipo y pudimos conversar con dos médicas.

En las entrevistas se detalló que los equipos estaban conformados de la siguiente manera: el Allende (2019) contaba con una médica especialista en fertilidad y sexóloga, a veces rotaban los residentes, y cuando lo necesitaban solicitaban informe a una asistente social. El equipo del Penna (2019) estaba compuesto por dos médicas, un médico, una asistente social, una psicóloga, una bióloga y una enfermera. Y el equipo del San Martín (2019) estaba formado por tres doctoras, dos médicas, un andrólogo, una bióloga y el ecografista.

\section{Resultados y discusión del estudio}

\section{Resultados sobre las técnicas de reproducción humana asistida en la salud pública.}

Pudo apreciarse que dentro de las TRHA existen dos tipos: los de baja y los de alta complejidad. Al respecto, la médica del hospital de Mar del Plata (abril de 2019) nos explicó: 
El tratamiento de baja complejidad consiste en pedir los laboratorios y hacer el diagnóstico. Se capacitó a un biólogo para que pudiera centrifugar el semen para hacer la preparación, y después poder nosotros hacer una inseminación mediante una cánula, ya que teníamos la medicación que hay que darle a la mujer para lo que es la inducción de la ovulación. Sino la otra opción de baja complejidad que se puede hacer, es el coito programado, que es lo que hacemos cuando no pueden pagar la preparación del semen. Nosotros programamos la inducción de la ovulación con la medicación que compra la paciente y, cuando está a punto de ovular, le aplicamos unas inyecciones y le hacemos tener relaciones.

Por su parte, los de alta complejidad precisan todo lo anterior, pero, además, en muchas ocasiones un banco de esperma y una sala de cirugía, para realizar la intervención de la unión del óvulo con el espermatozoide en el laboratorio y después la implantación en el cuerpo de la mujer. En esos casos, al principio en Mar del Plata, se lo tercerizaba en una clínica privada y el Ministerio de Salud provincial pagaba dos o tres tratamientos por mes. Al momento de la entrevista (abril del 2019), no había reactivo para preparar el semen, ni medicación para inducir la ovulación, así que solo podían realizar tratamientos de baja complejidad si las parejas podían pagar la medicación. En los casos de alta complejidad se los enviaba al hospital de La Plata que todavía conservaba el convenio con un banco de esperma privado.

En el hospital marplatense se realizaban seis consultas en el único día de la semana que atendían; si bien no nos dijeron el número de tratamientos que se llevaban a cabo por año, inferimos un estimativo de 288 . La médica sostenía que, en los tratamientos de alta complejidad, estadísticamente llegaban al embarazo entre el 8 y el 10\%. Los de alta complejidad se derivaban a La Plata donde debían contar con un mes de estadía en ese lugar para realizar el tratamiento. Asimismo, nos explicaba que recibían consultas de parejas, que lo podían hacer de manera privada, pero planteaban sus dudas en el hospital público, porque muchos pensaban que "lo de la fertilidad es un comercio". Dicha representación es socialmente difundida y se encuentra enmarcada en la forma en que se piensa la salud. Es decir, se sigue pensando que la fertilidad no es un tema de salud pública gratuita y por ello en su mayoría se deriva al sector privado. Si bien, desde el Ministerio de Salud de la provincia no se garantizan insumos e infraestructura, los profesionales de dichos servicios tampoco reclaman en función de la exigibilidad de las leyes, sino que los derivan a lo privado.

Cuando preguntamos acerca de la atención a parejas diversas, desde el hospital de Mar del Plata nos respondieron que recibieron consultas y nos ejemplificaron con un caso de una persona trans masculino. Dicha persona solicitó asesoramiento en relación con el tratamiento hormonal, el control de mamas y el PAP, donde se le advirtió que podía preservar su fertilidad antes de hacer el tratamiento. A este caso se suma otro, de una pareja de una mujer y un trans masculino, en el que ella llevó adelante el tratamiento a partir de comprar el semen de un donante privado, y que se encontraba, en esos momentos, cursando su embarazo. 
En el hospital de Bahía Blanca recibían entre 1000 y 1400 consultas por año, y en el 2018 habían realizado 70 tratamientos de alta complejidad y de baja complejidad un poco más. A estos últimos los hacen con autorización del Ministerio de Salud. De todas maneras, en el momento de la entrevista no se estaban realizando tratamientos de alta complejidad porque la sala de cirugía estaba fuera de servicio.

El hospital de Bahía Blanca contaba con banco de esperma público para pacientes oncológicos, para que puedan hacer una guarda previa a los tratamientos quimioterapéuticos, pero no se utiliza para otros casos. En este hospital, con respecto a la consulta de parejas diversas plantearon que "es bajo el porcentaje, es de mujeres, no hemos tenido de hombres y como no hay banco público, se las asesora para derivarlas a un centro privado" (médica del hospital Penna, 2019). Podríamos decir, que las derivaciones convalidan lo anteriormente mencionado: que la fertilidad es un comercio.

Si bien se insistió sobre el número de casos, no lo pudieron establecer. Esta fue una de las características de las tres entrevistas, la falta de información estadística, cuando sabemos que dichos datos sirven para exigir políticas públicas y demás. La falta de información estadística también puede denotar la falta de importancia que se le otorga a las cuestiones de fertilidad.

En el hospital de La Plata se realizaban 3000 consultas por año. Tampoco contaban con el número exacto de tratamientos llevados a término. Las médicas dicen que realizaron diez en un año a parejas de mujeres, pero ninguna de ellas pudo concebir. También relataban la atención a una pareja de mujeres en la que "él tiene cambio de género en el documento, es mujer" (médica del hospital San Martín, 2019).

En reglas generales encontramos que, desde que existe la ley de 2010 hasta el 2019 que se realizaron las entrevistas, si bien existieron consultas de parejas diversas, por un lado, las entrevistadas fueron inespecíficas en los datos estadísticos y, por el otro, solo encontramos un caso que terminó en embarazo en el hospital de Mar del Plata pero que fue la pareja quien aportó la muestra de semen comprada en lo privado. En los hospitales de Mar del Plata y de Bahía Blanca eran las parejas las que debían aportar el dinero para la compra del esperma, y en los casos donde se pudo realizar de manera pública, como en La Plata, ninguno fue exitoso.

\section{Los resultados relacionados con la adopción}

En cuanto al Poder Judicial, entrevistamos a una profesional del equipo de uno de los juzgados de La Plata donde está el Registro de Adoptantes. El juez a cargo, en los 90, fue el defensor de Mariela, una mujer trans que se quedó a cargo de los hijos de una vecina y que el barrio la denunció para que les sacaran a los niños, por el interés superior de los mismos; y finalmente, después del proceso jurídico, le dieron la adopción a Mariela. Enunciamos esta característica porque da cuenta que el juez no presentó ningún inconveniente al momento de la adopción por parejas diversas.

La psicóloga del equipo del juzgado comentaba que antes de la existencia de la ley (LNMI, 2010) se presentaban personas solas a adoptar y que hubo un caso del 2009 
en el que el juez solicitó saber si era realmente una mujer sola o si estaba en pareja con otra. Según la profesional, "no es lo mismo dos que uno, por las posibilidades, por la seguridad". En ese caso, la mujer afirmó que estaba en pareja hacía 17 años con otra y se llevó a cabo la adopción, en ese momento a nombre de una sola porque la ley solo lo permitía de esa manera.

La psicóloga sostiene que desde que la ley (LNMI, 2010) entró en vigencia, ninguna persona más se presentó sola si estaba en pareja con alguien del mismo sexo. Aclaró que la participación del juez en una organización llamada "Mami Chula" agilizó la llegada de parejas diversas, porque se sortearon los mitos en relación con la discriminación que se sufría en los juzgados. De todas maneras, la psicóloga contó que antes de su promulgación debían preguntar acerca de la orientación sexual de los aspirantes, no para discriminarlos, aunque funcionaba imaginariamente como tal. En general, las personas ocultaban su orientación sexual si no eran heterosexuales.

Resulta interesante que, si bien la psicóloga afirmó que no incidía la diferencia entre parejas de varones y parejas de mujeres para la adopción, para ella existían diferencias ancladas en que las mujeres tienen doble chance al poder acceder a un tratamiento de fertilización. Además, estableció una diferencia entre las parejas heterosexuales y las homosexuales; dijo que, por un lado, las segundas están más preparadas para un umbral más alto de frustración, porque vienen de muchos años de no acceder a la parentalidad. Por otro lado, las parejas homosexuales no juzgaban a la familia de origen porque "tienen muchísimo más acercamiento al dolor del otro, a la posibilidad de empatizar con el niño" (psicóloga del juzgado, 2019).

De todas maneras, la única pareja que entrevistamos durante la investigación (20162017), que adoptó a una niña recién nacida, si bien fue luego de la aprobación de la ley de Matrimonio Igualitario, explicó lo siguiente: "El matrimonio igualitario ya estaba cuando yo pido la adopción plena, sería 2011, 2012, o por ahí. Y teníamos todo planificado así y salió bien, muy bien la verdad. Pasé todas las entrevistas con cara de chapa sin mentir" (Luisa, 2019); "Omitiendo (risas)" (Carmen, 2019). El miedo a que pudiera incidir en la adopción las hizo reservar el dato de que estaba en pareja hacia muchísimo tiempo.

\section{Discusión}

Desde nuestra investigación, sostenemos que el acceso a la parentalidad, sea por TRHA, sea por adopción es una temática de Salud Mental, si la entendemos como la define la ley:

(...) como un proceso determinado por componentes históricos, socioeconómicos, culturales, biológicos y psicológicos, cuya preservación y mejoramiento implica una dinámica de construcción social vinculada a la concreción de los derechos humanos y sociales de toda persona (Ley Nacional Salud Mental, 2010, $\$$ Art. 3). 
A partir de esta definición podemos sostener que el acceso a la parentalidad no sólo es la concreción de un derecho humano, sino que también supone padecimiento psíquico de las personas que lo proyectan y no lo consiguen. Por ello no se puede concebir que, por cuestiones económicas, como supone poder pagar una TRHA en el ámbito de la salud privada, las personas no puedan acceder a la parentalidad. Debe ser el Estado mediante las políticas públicas enmarcadas en las leyes quienes garanticen dicho derecho.

Pudo verificarse en el estudio lo que sostiene el corpus acerca de que la atención en salud mental es altamente desigual en la Argentina y se asienta en la noción de enfermedad (Zaldúa et al., 2016). A eso se suma, en el caso de las mujeres, por la desigualdad de clase social, de etnia, de trabajo, de edad, de lugar de residencia, de racismo, de sexismo y de otros sistemas de opresión y discriminación. Una de las temáticas que según las autoras necesita urgente atención, es la decisión de las mujeres sobre sus derechos sexuales y reproductivos. Si bien en este marco se reclamaba la legalización del aborto, también es necesario acompañar a las mujeres en el momento de la reproducción si ella no se desarrolla de la manera natural esperada.

$\mathrm{Al}$ entrevistar, durante el 2015, a la trabajadora social del equipo del hospital de La Plata sobre la Ley Provincial de Reconocimiento de la Infertilidad Humana como enfermedad (LPRIHE, 2010) nos dijo: "De hecho hasta muy enojada estaba porque decía no es un problema de salud pública, es más grave la tuberculosis, la diabetes, como problema de salud pública" (trabajadora social hospital San Martín, 2015).

Asimismo, existen ONG que colaboran para que estos derechos sean posibles. Sin embargo, una de las frases que nos llamó la atención, es la que dijo la abogada de una ONG, en diálogo con nuestro equipo (2019), acerca de la salud pública:

El que no tiene plata siempre tiene que pelear en este país para acceder a lo que sea de salud pública. Siempre pasa eso en todo sentido y más en esto que no es una cuestión de salud, sino de querer tener hijos.

Es decir que, para la trabajadora social, el hecho de no poder acceder a la parentalidad y sus consecuencias sobre la salud mental de una pareja, no era considerado como un tema de salud. En el mismo sentido, la abogada de la ONG lo pensaba como un derecho, pero no como un tema de salud pública.

En este espacio, es necesario pensar la situación de las parejas diversas, que proyectan un/a hijo/a pero no cuentan con los medios económicos necesarios. Si bien la Ley Nacional de Reproducción Médicamente Asistida 26.682 (LNRMA, 2013) estableció el derecho de acceder a un TRHA sin que los gametos sean de la pareja, en tres de los cuatro hospitales de la provincia que fueron elegidos para llevar adelante dicha política pública, no contaban con banco de donantes públicos; en los casos donde se le puede solicitar al Ministerio de Salud que pague las muestras, en el momento de la 
entrevista no tenían sala de cirugía para realizar el procedimiento, por ejemplo, en el hospital de Bahía Blanca.

Más allá de la política de vaciamiento de los servicios de salud pública durante los años 2015-2019, que podrían explicar dicha situación, encontramos solo una mujer lesbiana que pudo acceder de manera gratuita a un embarazo mediante el procedimiento en Mar del Plata. Sin embargo, ella tuvo que comprar la muestra de esperma, es decir, que esta ley de Fertilidad Asistida (LPRIHE, 2010) no estaría cubriendo las necesidades de este sector de la sociedad.

Retomamos el concepto que proponen Zaldúa et al. (2016), cuando hablaban de desigualdad en salud, que refiere a las distintas oportunidades y recursos relacionados con la salud según la clase social, su género, su territorio o su etnia. En las entrevistas nos decían que cuando recién surgió la ley provincial en el 2010, consultaban parejas de todas las clases sociales.

En el 2013 la ley nacional (LNRMA, 2013), obligó a las obras sociales a costear los tratamientos. En ese momento las consultas en los hospitales públicos se convirtieron en su mayoría de sectores sociales empobrecidos, excepto en Mar del Plata. En dicho hospital llegaban parejas para hacer una interconsulta, pero el tratamiento lo hacían en privado. Dicho comportamiento responde al hecho que muchas parejas consideran las TRHA como un comercio, según los profesionales.

En las entrevistas realizadas a parejas de mujeres con hijos/as, o proyecto de hijos/ as, en la investigación realizada por el mismo equipo durante los años 2016-2017, nos encontramos con dos atravesamientos: de clase y de género. Quienes no contaban con obra social o el dinero suficiente para llevar adelante un TRHA, ni siquiera consultaban en los hospitales públicos. Es a este sector donde no llegaba esta política pública. Esto hace que muchas parejas de mujeres lo estuvieran resolviendo de "manera casera": contactaban a un conocido, o desconocido, que pudiera convertirse en donante y realizaban el procedimiento con una pipeta en domicilio. El problema de dicho procedimiento es el reconocimiento legal de la otra mujer de la pareja como madre de ese niño/a, ya que para el Registro Civil no tiene ninguna participación porque no se firmó la voluntad procreacional en el centro de reproducción, tal como lo exige la ley (LNMI, 2010). De todas maneras, existen casos que lograron que la madre social (es decir quien no lleva adelante el embarazo) sea reconocida como tal ante el Registro Civil, a partir de la organización Abogadxs por los Derechos Sexuales (ABOSEX, 2017).

En términos jurídicos la voluntad procreacional es determinante de la filiación de la madre social sostienen Iturburu et al. (2016) que dicha voluntad debe ser manifestada en el consentimiento previo, informado y libre, que se realiza donde se llevan a cabo los TRHA y, está claro para la ley (LNMI, 2010), que es independiente de quien aporte los gametos y el cuerpo que lleva adelante el embarazo.

Según plantean Zaldúa et al. (2016), la resolución de la accesibilidad y de la equidad se pueden garantizar con la participación comunitaria y con los escenarios territoriales, pasar de ser objetos a sujetos e instalarse como protagonistas de sus decisiones. 
En función de lo anterior, entrevistamos al equipo del Programa de Extensión "Diversidad Familiar y Derecho de Familia", a cargo de la abogada Lópes -Facultad de Ciencias Sociales y Jurídicas, UNLP-. Ellos nos contaron que acompañan en diferentes situaciones que no cuentan con resolución e intentan armar estrategias con presentaciones legales para acceder a dicho derecho.

En esta misma línea, dialogamos con la abogada de una organización LGTBIIQ de La Plata, quien nos relató situaciones que se le presentaban y cómo las resolvían. Explicó la diferencia entre las parejas de heterosexuales y las diversas, en los casos de "inseminación casera". Si una mujer se queda embarazada, cuando nace el/la hijo/a va al Registro Civil, el padre da su apellido y ahí culmina el trámite. Si va una pareja de mujeres sí o sí tiene que llevar la firma del papel de la voluntad procreacional que les otorga el centro de salud. Pero si ellas no tienen el certificado porque hicieron la inseminación en su casa, esta situación establecería diferencias entre ambos tipos de parejas, ya que, como no podrían realizar el trámite, se perdería la igualdad jurídica que sostiene el Código Civil, a partir de la reforma, con la Ley de Matrimonio Igualitario (2010).

Asimismo, visualizamos las dificultades en la exigibilidad, en los casos presentados de parejas diversas con falta de recursos económicos, desconocimiento de derechos en general y de la salud sexual y reproductiva, de la misma manera que en las comunas de salud estudiadas en el trabajo mencionado de Zaldúa et al. (2016).

La epidemiología crítica de Breilh (2013) plantea una comprensión dialéctica del proceso de determinación por generación y reproducción, con ello se incorporan las nociones de proceso y de lo colectivo. Asume los procesos colectivos o grupales como elementos irreductibles a lo individual y como espacio de intervención. La salud no obedece a un orden exclusivamente individual, es necesario pensarlo como un proceso complejo y socialmente determinado.

En las entrevistas realizadas, encontramos que una de las médicas relata que en la mayoría de los casos los problemas de la reproducción se encuentran ligados a que las mujeres de los sectores medios, comienzan a buscar un embarazo pasados los 35 años, cuando la edad más fértil es entre los 20 y los 30. Planteado de esta manera podría ser un problema individual, pero desde una perspectiva de género y social, sabemos que la llegada de un/a niño/a a una familia detiene, o retrasa, el desarrollo profesional de las mujeres. Entonces, muchas prefieren terminar sus estudios y esperar a obtener un trabajo estable para empezar a buscar un/a hijo/a.

En las parejas heterosexuales existe una división sexual del trabajo entre lo público y lo privado (afuera y adentro). Es necesario rescatar que la división del afuera y del adentro tuvo consecuencias sobre el desarrollo laboral y profesional de las mujeres a lo largo de la historia. En una nota del diario Página 12, del 4 de noviembre de 2016, se confirmó que los puestos jerárquicos del CONICET, solo están ocupados en un $25 \%$ por mujeres, que muchas veces son parejas que comparten disciplinas y el ingreso al organismo. Cuando llegan los/las hijos/as, el varón continúa ascendiendo y la mujer sufre una detención de su carrera laboral por causa del embarazo, de los 
primeros cuidados, de la asistencia a los/las hijos/as en situaciones de enfermedad, entre otras cuestiones.

En este momento, volvemos sobre la temática analizada, el derecho al acceso a la parentalidad por técnicas de reproducción humana asistida para las parejas de mujeres, que, si bien se traduce en leyes, no se cumplen, ya sea por falta de recursos o porque ciertos profesionales -como la trabajadora social de uno de los hospitalesno piensan que sea un problema de salud importante.

Para acompañar la exigibilidad del derecho al acceso a la parentalidad, se otorgaban diferentes soluciones, entre ellas la del "semen solidario", que consiste en un sistema que se encuentra en páginas de Facebook, de hombres que ofrecen su semen de manera solidaria, es decir sin costos económicos, ni participación en la parentalidad. Si una pareja de mujeres resuelve buscar dicha solución y llevar adelante una inseminación casera, se puede presentar un amparo judicial para que la madre no gestante pueda serlo en la inscripción del niño/a en el Registro Civil. En Argentina, un amparo judicial es un pedido que se le hace al juez para defender los derechos fundamentales de las personas.

La abogada de la ONG entrevistada planteó que no conocía personas de la comunidad LGTTBIIQ que hubieran realizado tratamientos en hospitales públicos por la falta de información. Si bien no lo reconoce como un tema de salud, como lo mencionamos anteriormente, sí lo entiende como un tema jurídico, ya que en la institución en la que ella participa acompañan jurídicamente a parejas diversas en los trámites necesarios para acceder a la parentalidad. La abogada comentó que se encontraba siguiendo un caso que había enviado al Colegio de Abogados de la ciudad de La Plata quienes le contestaron: "No trabajamos la Ley de Matrimonio Igualitario". Este es punto de controversia, ya que dicha ley está incorporada al Código Civil y ningún abogado puede trabajar por fuera del mismo. Con relación a la adopción es necesario plantear como lo propone el Código Civil y Comercial en la Argentina:

La adopción es una institución jurídica que tiene por objeto proteger el derecho de niños, niñas y adolescentes a vivir y desarrollarse en una familia que le procure los cuidados tendientes a satisfacer sus necesidades afectivas y materiales, cuando éstos no le pueden ser proporcionados por su familia de origen. (Código Civil y Comercial, 2015, $\$$ Art. 594)

Algunas de las precauciones que toman ciertas entidades oficiales, operan para que el/la niño/a no vuelva a tener situaciones de tristeza. Entre los requisitos para la adopción están: a) tener 25 años de edad, salvo que el cónyuge cumpla este requisito; b) diferencia de edad entre adoptante y adoptado de 16 años; c) residencia permanente en el país por un mínimo de 5 años y e) estar inscripto en el registro de adoptantes.

Existen tres tipos de adopción: la plena, la simple y la de integración, y la elección entre las tres se establece por el principio del interés superior del niño/a. En la plena se le otorga al adoptado la condición de hijo/a y desaparecen los vínculos jurídicos con su familia de origen. La simple confiere el estado de hijo/a adoptado/a, pero no 
crea vínculos jurídicos con la familia de origen ni con el/la cónyuge del adoptante. En la de integración, se adopta al hijo/a del cónyuge o del conviviente.

En su escrito, Lópes (2018) plantea que el código solo contempla para la adopción el proyecto parental de pareja y no de otras relaciones entre adultos como, por ejemplo, de amistad. Es necesario remarcar que en la actualidad la adopción es "casi" la única manera en que las parejas de varones puedan acceder a la parentalidad en el país. Decimos "casi", porque existen algunos casos de subrogación de vientre (Vidal, 2019).

A partir de la reforma del Código Civil, se permite que los adoptantes puedan inscribirse como integrantes de la unión convivencial -sean del mismo o de diferente sexo-, antes solo podían hacerlo en el Registro de Adoptantes cuando eran cónyuges. En Argentina, uno de los problemas de la adopción es que la mayoría de los interesados solicitan niños/as menores de un año y la mayoría de los que están en estado de adoptabilidad son más grandes, dado que la justicia se toma el tiempo necesario para evaluar si separa al niño/a de su familia biológica.

Tomaello y Russomando (2011), a partir de un estudio con parejas o personas adoptantes, señalan los diferentes motivos para adoptar que encontraron: proyecto, perpetuación, miedo a la vejez, llenar vacíos, desafío, cumplir mandatos, realización personal y todas a su edad son madres. Dichos motivos son tenidos en cuenta en el momento de la adopción porque influirán en la vida posterior del niño/a. Las autoras explican que la ley permite que las parejas homosexuales adopten pero que este proceso sólo se efectivizará si los profesionales dejan de lado los prejuicios de dudar o de pensar que no es bueno darle un/a niño/a en adopción.

Spivacow (2011) plantea que muchas veces los profesionales consultan si es conveniente dar o no un/a niño/a en adopción a homosexuales. El autor no cree que los psicoanalistas solos puedan dar la palabra científica y certera, pero que al formar parte de equipos interdisciplinarios sí tendrían mucho para aportar (Spivacow, 2011). Algunos profesionales que legislan sobre el amor, la pareja y la familia, lo hacen desde un lugar de autoridad y ése no es el del psicoanálisis.

En su texto, Tarducci (2011) analiza las buenas y las malas madres de la adopción. El artículo examina el hecho puntual de la provincia de Misiones, donde se dan adopciones directas, que consisten en poner en relación dos grupos de parentesco que no deberían conocerse: uno cuenta con una mujer embarazada y el otro con intenciones de adoptar. Termina siendo un acuerdo entre particulares que luego resulta avalado por un juzgado, es decir que técnicamente, es legal. Sin embargo, este tipo de adopción, en donde los adoptantes y la madre biológica se conocen de antemano, está prohibida por el Código Civil.

En la mayoría de los casos, los/as niños/as son dados/as en adopción por situaciones de pobreza. En este sentido, Tarducci (2011) sostiene que la adopción no puede ser una política social antipobreza y que, en estos casos, el Estado podría implementar políticas de acompañamiento para las familias carenciadas, y no solamente fomentar que los/as niños/as sean dados en adopción. 
Las buenas madres son aquellas que le dan un hogar y una familia al niño/a y que de alguna manera realzan los valores simbólicos de la maternidad. Las malas madres son las que dan sus hijos/as en adopción, las que incuban a sus hijos/as y le dan lugar al negocio. Son los medios de comunicación y los juzgados los que sostienen desde el discurso social las valoraciones de buenas y malas, pero no lo que exponen así ni Tarducci (2011), ni el equipo de esta investigación.

En los informes socio-ambientales que realizan las trabajadoras sociales de los juzgados de familia para evaluar la situación de quien va a entregar un/a niño/a en adopción, se menciona entre los motivos a "serios problemas económicos" (Tarducci, 2011). Asimismo, relatan que el hecho de entregarlos hace que las madres biológicas sean juzgadas, incluso desde el entorno donde viven.

En relación con las buenas madres, deben responder al ideal que esta sociedad asigna para ser mujer-madre, es decir, estar casadas con maridos que tengan un buen empleo, que no sean muy ansiosas y si son católicas, mejor.

En una nota de diario titulada "Los homosexuales son menos exigentes a la hora de adoptar" (Lalaurette, 2014), se mostró que los/as homosexuales son once veces más propensos a aceptar la guarda de niños/as entre 6 y 10 años de edad que los heterosexuales, según los datos que maneja el Registro Único de Aspirantes a guarda con Fines Adoptivos que depende del Ministerio de Justicia y Derechos Humanos de la Nación. Asimismo, el 8,5\% de los/as aspirantes homosexuales están dispuestos a adoptar niños/as de estas edades con patologías leves o graves, y esa proporción no se repite en la generalidad de los/as aspirantes (Lalaurette, 2014).

En la nota es consultada Lópes, quien expone diferencias entre las parejas homosexuales y las heterosexuales. Según la abogada, las parejas homosexuales observan la adopción desde su verdadera función, es decir, darle una familia a un/a chico/a, mientras que, en las parejas heterosexuales, la adopción significa obtener un hijo/a para la pareja, en el contexto de visualizar la maternidad como sinónimo de completitud (Lalaurette, 2014).

En la misma nota, Paradiso (como se citó en Lalaurette, 2014), titular del área jurídica de la Comunidad Homosexual Argentina (CHA), afirma que para los/ as homosexuales no existe la presión social de tener hijos/as para cumplir con los modelos y mandatos sociales, y advierte que, el hecho de que sean más inclusivos/as, no tiene que hacer que se conviertan en familias residuales, o sea donde vayan los/as niños/as que el resto no acepta.

A partir de los datos de la Dirección Nacional de Registro de Aspirantes a Guarda con Fines Adoptivos, el total de inscriptos fue de 7034, de los cuales 60 pertenecen a matrimonios igualitarios, es decir que están legalmente casados, y once son a parejas igualitarias. En relación con la disponibilidad adoptiva respecto de la edad del/a niño/a, en general, el 92,23\% acepta de hasta un año, el 27,45 \%, de hasta seis y el 1,01\%, de hasta diez. En cambio, en el caso de aspirantes homosexuales, el 88,73\% acepta de hasta un año, el 53,52\%, de hasta seis, y el 11,27\%, de hasta diez (Lalaurette, 2014). 
Paradiso (como se citó en Lalaurette, 2014) asegura que, si bien desde las parejas homosexuales casadas pueden presentarse como aspirantes a adoptar, en el Poder Judicial continúan eligiendo al matrimonio heterosexual, pues la mayoría del sistema judicial es homofóbico.

Existen prejuicios en la sociedad en general y según cuenta Paradiso (como se citó en Lalaurette, 2014), hace un tiempo cuando le otorgaron la guarda de unos niños a una pareja gay, los vecinos realizaron denuncias alegando que los niños estaban en peligro. En esta línea, Raíces Montero (2012) sostiene que desde las religiones fundamentalistas, filosofías esencialistas, ciencias médicas obsoletas y ciertas corrientes psicológicas pseudo ortodoxas, la moral y el sentido nocional que se basa en prejuicios, preconcepciones o apriorismos puros, desde el Estado y el discurso médico psiquiátrico se promueve la heterosexualidad estable y procreativa como la única sexualidad normal. Dichas instituciones presentan a otras formas de sexualidad como anormales, patológicas e irresponsables.

Raíces Montero (2012) sostiene que negarles la adopción a parejas del mismo sexo se apoya en la sobrevaloración de la heterosexualidad y así se propone a la familia heteronormativa y patriarcal. Se plantea la idea de que los roles deben ser cumplidos por los padres y por las madres, vinculados a la diferencia de sexo, y es por eso que los homosexuales no pueden adoptar, ya que si son por ejemplo dos varones no estarían cumplidos los dos roles el masculino y el femenino. En estudios anteriores (Vidal, 2012) hemos trabajado sobre como entendemos desde el equipo las funciones: de amparo y de límite, donde no es necesario que sean cumplidos por mujeres o varones biológicamente entendidos.

Pensamos que no es necesario aclarar que, en el seno de esta investigación, sostenemos que los roles no están atados al sexo de quien lo ejerce y que existen numerosos estudios que muestran la misma capacidad, tanto de homosexuales como de heterosexuales, para educar adecuadamente a niños y niñas en adopción. Es por ello que creemos no deben ser tratadas de un modo diferente ambos tipos de familia por el Poder Judicial. La elección de los padres adoptantes debe ser en función de sus habilidades parentales y la orientación sexual o la identidad de género no deben ser factores excluyentes para valorar la idoneidad. En este sentido, Bigliardi (2017), especialista en derecho de familia de la Universidad Nacional de La Plata, sostiene que las parejas homosexuales y las heterosexuales cuentan con los mismos derechos frente a la adopción, pero las representaciones de lo que es una familia, en general pensada desde una lógica heteronormativa, de los operadores judiciales no contribuyen a cumplir con la igualdad.

En el mismo sentido, en la entrevista realizada en el Proyecto de Investigación 2016-2017, por nuestro equipo, muestra como una pareja de mujeres prefiere omitir su relación sexo-afectiva en el proyecto de adopción de una niña, por temor a los prejuicios de los integrantes del Juzgado de Familia.

En una nota de la Revista Noticias, publicada en marzo de 2017, el 2\% de los matrimonios o parejas igualitarias fueron parte del Registro Único de Aspirantes 
a Guarda con fines Adoptivos (RUAGA). Al consultar a María Rachid, directora del Instituto contra la Discriminación de la Ciudad de Buenos Aires, y a Andrea Rivas, abogada y presidente de la Asociación Familias Diversas (García Testa, 2017), opinaron que la baja proporción se debe a una discriminación internalizada que se suma el prejuicio de la justicia, y que por ello las parejas diversas prefieren elegir otras vías como la inseminación casera o la subrogación de vientre.

Sin embargo, a partir de las notas de los tres diarios (La Nación, Página 12 y Revista Noticias), se encontró que los profesionales cometen prácticas discriminatorias en sus evaluaciones e informes cuando se refieren a parejas homosexuales -tanto de varones como de mujeres-, sin basarse en un conocimiento científico y profesional.

\section{Conclusión}

Como vimos hasta aquí, si bien se avanzó muchísimo en materia legal respecto del acceso a derechos para los grupos diversos, dichas leyes no se visibilizan en las prácticas. Es decir, que la desigualdad sigue existiendo y la exigibilidad, si bien cuenta con sectores que se movilizan hacia cambios y que logran por momentos sortear los obstáculos, en otros períodos terminan reproduciendo el antiguo paradigma, cuando piensan que el acceso a la parentalidad no es una cuestión de salud.

Continúan existiendo resistencias al acceso al derecho de parentalidad, ya sea por falta de insumos o por falta de infraestructura. Podemos sostener que las profesionales de la salud entrevistadas se mostraron predispuestas a acompañar el proceso de acceso a la parentalidad y ninguna manifestó resistencias a ello. Sin embargo, no encontramos puntos de resistencia ni procesos de exigibilidad hacia las políticas públicas en relación con las carencias con las que cuentan.

Tampoco encontramos movimientos de exigibilidad desde las parejas de mujeres. La totalidad de las entrevistadas durante la investigación anterior (2016-2017) acudieron a centros de reproducción privados. Si bien entendemos que todo el movimiento es esperable realizarlo en un encuadre cuidado, el hecho de no solicitarlo en la salud pública no sienta precedente como una demanda "real" de tratamientos en estas parejas.

Se sigue sosteniendo las TRHA desde lo privado, sea desde la cobertura de la obra social o de manera particular. Los mismos equipos de los hospitales realizan las derivaciones a centros privados al no contar con banco de esperma público o sala de cirugía para realizar la práctica. De alguna manera, se sigue sosteniendo aquello de que la fertilidad es un negocio, ya que solamente se realiza en sectores privados. En futuras investigaciones, se podría indagar acerca de cómo piensan los profesionales de la salud los TRHA, es decir si es o no para ellos, un tema de salud prioritario.

Es necesario resaltar la importancia del rol del financiamiento de las políticas públicas porque, como visualizamos en las entrevistas, si bien la demanda es poca, existe -los profesionales idóneos también- pero sigue sin crearse un banco de donantes público que incidiría en el cumplimiento de los derechos proclamados por las leyes 
provincial (LPRIHE, 2010) y nacional (LNRMA, 2013) que enmarcan la infertilidad humana y el acceso a la parentalidad encuadrada en la ley de Matrimonio Igualitario.

Desde la investigación accedimos a actores del Poder Judicial que manifestaron no contar con prejuicios y trabas al momento de dar en adopción un/a niño/a a una pareja diversa. Sin embargo, abogados y representantes de organizaciones que trabajan por los derechos de la comunidad LGTTBIQ, sostienen que siguen encontrándose dificultades a la hora de que las parejas diversas puedan adoptar. Los equipos de los Juzgados siguen prefiriendo las parejas heterosexuales, a partir de un marco heteronormativo, para otorgar la adopción de un niño/a.

En relación con las limitaciones de la investigación, como accedimos a un solo miembro de cada institución no hemos podido profundizar acerca de las significaciones sociales imaginarias del conjunto de los equipos de salud, así como del poder judicial. Dicha tarea quedará como futuras líneas de investigación.

El acceso al derecho a la parentalidad sostenemos que: queda mucho camino por recorrer.

\section{Agradecimientos}

Al financiamiento de Ciencia y Técnica de la Universidad Nacional de La Plata, en tanto Proyectos Promocionales de Investigación y Desarrollo (2018-2019: Código S018).

A los integrantes del equipo de investigación que durante estos dos años colaboraron con el armado del cuadro teórico mediante discusiones y diálogos rigurosos, así como en la realización, desgrabación y análisis de las entrevistas. Mi más sentido agradecimiento a: Belén Alfonso, Federico Batiz, Magdalena Gurini, Paula Iparraguirre, Manuela Kreiss, Mariela Pantano, Federico Prieto Courries y Celeste Ruiz.

Asimismo, a todos/as y cada uno/a de los integrantes de los equipos de salud del Poder Judicial, equipos de Extensión Universitaria e integrantes de ONG que nos brindaron su tiempo para contarnos acerca de su experiencia en la temática sus reflexiones al respecto. 


\section{Referencias}

Abogadxs por los Derechos Sexuales (2017). ABOSEX logró la inscripción registral desjucializada de un reconocimiento de co-maternidad por técnica de reproducción asistida “casera". HTTPS:// ABOSEX.COM.AR/2017/10/30/ABOSEX-LOGRO-LAINSCRIPCION-REGISTRAL-DESJUDICIALIZADADE-UN-RECONOCIMIENTO-DE-CO-MATERNIDADPOR-TECNICA-DE-REPRODUCCION-ASISTIDACASERA/

Bigliardi, K. (2017). Las barreras ocultas que sufren las parejas gay para adoptar. Revista Noticias. HTTPS://NOTICIAS.PERFIL.COM/2017/03/10/LASBARRERAS-OCULTAS-QUE-SUFREN-LAS-PAREJASGAY-PARA-ADOPTAR-UN-HIJO/

Birulés, F. (2008). El género es extramoral. Entrevista con Judith Butler. Barcelona Metrópolis, 72, s/p.

Blanchet, A. y Gotman, A. (1992). L'enquête et ses méthodes: l'entretien. Nathan Univeristé.

Breilh, J. (2013). La determinación social de la salud como herramienta de transformación hacia una nueva salud pública (salud colectiva). Revista Facultad Nacional de Salud Pública, 31, 13-27.

Código Civil y Comercial Ley $\mathrm{n}^{\circ}$ 26.994, República Argentina (2014). HTTP://SERVICIOS. INFOLEG.GOB.AR/INFOLEGINTERNET/ ANEXOS/235000-239999/235975/NORMA.HTM

Etchichury, H. (2010). Los mismos derechos el mismo nombre. Revista Contingencia Matrimonio Igualitario, 3(7).

Fernández, A. M. (2006). La mujer de la ilusión. Pactos y contratos entre hombres y mujeres. Paidós.

Fernández, A. M. (13 de agosto de 2015). Nuevos modelos de familia "mujer-mujer". Página 12. HTTPS://WWW.PAGINA12.COM.AR/DIARIO/ PSICOLOGIA/9-279229-2015-08-13.HTML

García Testa, V. (10 de marzo de 2017). Las barreras ocultas que sufren las parejas gay para adoptar. Revista Noticias. HTTPS://NOTICIAS.PERFIL. COM/2017/03/10/LAS-BARRERAS-OCULTAS-QUESUFREN-LAS-PAREJAS-GAY-PARA-ADOPTAR-UNHIJO/

Hiller, R. (2010). Matrimonio igualitario y espacio público en Argentina. En L. Aldao Marin-Clérico (comp.), Matrimonio igualitario. Perspectivas sociales, políticas y jurídicas (pp. 85-130). Eudeba.

Iturburu M., Salituri Amezcua, M.y Vázquez Acatto M. (2016). La regulación de la filiación derivada de las técnicas de reproducción asistida en la Argentina: voluntad procreacional y consentimiento informado. Revista IUS, 11(39), 1-31.
Lalaurette, S. (18 de agosto de 2014). Los homosexuales son menos exigentes a la hora de adoptar. La Nación. HTTPS://WWW.LANACION. COM.AR/SOCIEDAD/LOS-HOMOSEXUALES-SONMENOS-EXIGENTES-A-LA-HORA-DE-ADOPTARNID1719017

Ley Nacional de Matrimonio Igualitario $\mathrm{n}^{\circ}$ 26.618, República Argentina (2010). нттр:// WWW.ACNUR.ORG/FILEADMIN/DOCUMENTOS/ BDL/2017/10957.PDF

Ley Nacional de Reproducción Médicamente Asistida. Acceso Integral a los procedimientos y tecinas médico-asistenciales de reproducción médicamente asistida $\mathrm{n}^{\circ}$ 26.682, República Argentina (2013). HTTP://SERVICIOS. INFOLEG.GOB.AR/INFOLEGINTERNET/ ANEXOS/215000-219999/216700/NORMA.HTM

Ley Nacional de Salud Mental no 2.6657, República Argentina (2010). HTTP://SERVICIOS. INFOLEG.GOB.A R/I N F OLEGIN T ER N E T/ ANEXOS/175000-179999/175977/NORMA.HTM

Ley Provincial Reconocimiento de la Infertilidad Humana como enfermedad. Reconocimiento de la cobertura médico asistencial integral de las prácticas médicas a través de las técnicas de fertilización asistida $n^{\circ} 14.208$, Provincia de Buenos Aires (2010). HTTP://WWW.ME.GOV.AR/ DOC_PDF/LEY14208.PDF

Lópes, C. (2018). Familias formadas por parejas del mismo sexo y el Código Civil y Comercial de la Nación. Derecho y Ciencias Sociales, 19, 22-44. HTTPS://DOI.ORG/10.24215/18522971E037

Página 12 (4 de noviembre de 2016). Platos sucios. Página 12. HTTPS://WWW.PAGINA12.COM.AR/ DIARIO/SUPLEMENTOS/LAS12/13-10993-2016-1104.HTML

Pecheny, M y De La Dehesa, M. (2010). Sexualidades y políticas en América Latina: un esbozo para la discusión. HTTP://WWW.SXPOLITICS.ORG/PTBR/ WP-CONTENT/UPLOADS/2009/10/SEXUALIDADESY-POLITICAS-EN-AMERICA-LATINA-RAFAEL-DELA-DEHESA-Y-MARIO-PECHENY.PDF

Raíces Montero, L. (9 de febrero de 2012). Adopción: la caída del prejuicio. Fundación Reflejos de Venezuela. HTtPs://WwW. FUNDACIONREFLEJOSDEVENEZUELA.COM/ NUEVA-FAMILIA/A DOPCIN-LA-CADA-DELPREJUICIO-LIC-JORGE-HORACIO-RACESMONTERO/

Spivacow, M. (2011). La pareja en conflicto. Paidós. 
Strauss, A.y Corbin, J. (2002). Bases dela investigación cualitativa. Técnicas y procedimientos para desarrollar la teoría fundamentada. Editorial Universidad de Antioquia.

Tarducci, M. (2011). Las "buenas" y "malas" madres de la adopción. En K. Felitti (coord.), Madre no hay una sola. Experiencias de maternidad en la Argentina (pp. 199-210). Ediciones CICCUS.

Tomaello, F. y Russomando M. (2011). Adopción. La construcción feliz de la paternidad. Paidós.

Vidal, I. (2012). Funciones de amparo y de límite en parejas del mismo sexo. Cuarto Congreso Internacional Psicología. Facultad de Psicología de la Universidad Nacional de La Plata.

Vidal, I. (15-16 de noviembre de 2018). Acceso a la parentalidad en parejas del mismo sexo $y$ su incidencia con el contexto social y familiar [Resumen de presentación de la conferencia]. Actas de las $6^{a}$ Jornadas de Investigación en Psicología y $5^{\circ}$ encuentro de Becarias, Becarios y
Tesistas. Facultad de Psicología de la Universidad Nacional de La Plata. HTTP://WWW.PSICO.UNLP. EDU.AR/UPLOADS/DOCS/MEMORIAS JORNADAS INVESTIGACION 20180709.PDF

Vidal, I (2019). La subrogación como vía de acceso a la parentalidad en parejas de varones en Argentina. Séptimo Congreso Internacional de Investigación en Psicología. Facultad de Psicología UNLP.

Zaldúa, G., Bottinelli, M., Longo, R, Sopransi, M. y Lenta, M. (2016). Exigibilidad y justiciabilidad desde la epidemiología territorial. Una construcción inicial con actores comunales. En Zaldúa, G. (coord.), Intervenciones en psicología social comunitaria: territorios, actores y políticas sociales (pp. 21-54). Teseo. 